\title{
La parole des prêtres, un outil de politisation révolutionnaire (Royaume des Deux-Siciles, 1799-1848)
}

Pierre-Marie Delpu (École des Hautes Études Hispaniques et Ibériques, Casa de Velázquez)

Après avoir été l'un des principaux vecteurs de la mobilisation populaire légitimiste qui a suivi la révolution napolitaine de 1799, la parole des prêtres s'est imposée, au cours du premier XIX ${ }^{\mathrm{e}}$ siècle, comme l'un des outils les plus efficaces de la politisation révolutionnaire, capable de convertir à la révolution des acteurs populaires qui lui étaient traditionnellement sinon opposés, du moins réticents. La place que les prêtres de village ont acquise parmi le personnel politique qu'on commence à qualifier de «libéral » contribue à l'expliquer: c'est à l'un d'entre eux, Matteo Farro, curé de Bellosguardo dans la province de Salerne, qu'on doit l'introduction de la constitution espagnole de Cadix au nom de laquelle a eu lieu la révolution de $1820-1821^{1}$. Ces prêtres constituent des intermédiaires décisifs du courant libéral auprès du peuple, à la tête d'un processus de politisation qui se construit pendant le premier XIX ${ }^{\mathrm{e}}$ siècle. Celui-ci articule des épisodes révolutionnaires nationaux $(1799,1820-1821,1848)$, des révoltes plus ponctuelles à rayonnement local (Pouilles, 1817 ; Cilento, 1828 ; Naples, 1833 ; Calabres, 1837 puis 1844 et 1847 ; Abruzzes, 1841) et des conjonctures plus fluides sans bouleversement politique majeur. Il voit s'amplifier la participation politique du peuple, de la «révolution passive» de 1799 menée par les élites à l'été 1848 où culmine la contestation populaire contre le roi Ferdinand II. Le phénomène a donc été progressif et a profité du rôle central qu'occupait alors la religion dans la vie sociale du royaume.

Si les prises de parole politiques des prêtres, dominées par la pratique de la prédication, y ont joué un rôle fondamental, elles ne sont propres ni au royaume des Deux-Siciles, ni au monde catholique ${ }^{2}$ : l'Europe méditerranéenne dans un sens large a vu s'affirmer des usages politiques nouveaux de la religion, destinée à accompagner l'apprentissage politique du peuple au tournant des XVIII e et XIX ${ }^{\mathrm{e}}$ siècles. Elle a fourni aux révolutionnaires des images, des outils pédagogiques, transférés dans l'ordre

1 Biblioteca Nazionale Centrale, Rome, Mis. Ris., f. 106. Dans le vocabulaire politique napolitain, l'adjectif « libéral » s'impose progressivement, dans la première moitié du $\mathrm{XIX}^{\mathrm{e}}$ siècle, pour désigner les partisans des libertés politiques et économiques. Sans constituer une option politique formalisée, ils se confondent globalement avec l'opposition de gauche aux Bourbons. Voir sur ce point Pierre-Marie Delpu, Un autre Risorgimento. La formation du monde libéral dans le royaume des Deux-Siciles (18151856), Rome, École française de Rome, 2019, p. 78.

2 Pour les pasteurs protestants français de la même époque, voir Céline Borello, $L a$ république en chaire protestante, XVIII ${ }^{e}-X I X^{e}$ siècles, Rennes, PUR, 2018. Sur la politisation des prêtres, voir par exemple Yann Raison du Cleuziou (dir.), La politisation des clercs (XIXe-XXe siècles), dossier spécial de Histoire, mondes et cultures religieuses, $\mathrm{n}^{\circ} 42,2017 / 2$. 
politique, et dont les prêtres ont été des passeurs de premier ordre ${ }^{3}$. La très forte religiosité populaire qui caractérise la société du Mezzogiorno depuis l'époque moderne l'inscrit résolument dans cette évolution. Les prédications révolutionnaires des prêtres ont permis de légitimer auprès du peuple la révolution et de l'intégrer à cette dernière. Elles constituent donc une voie privilégiée des « politisations ordinaires » qui ont permis d'imposer la révolution auprès des masses du royaume ${ }^{4}$.

\section{UNE PRATIQUE DE LA PEDAGOGIE POLITIQUE REVOLUTIONNAIRE}

L'utilisation politique de la parole des prêtres part d'un postulat: l'acceptation de la révolution par le peuple ne peut se faire qu'au terme d'un processus d'instruction des masses. Elle constitue l'une des formes ordinaires des pratiques d'éducation populaire qui se développent dans les premières décennies du XIX siècle et qui, dans l'espace italien, se focalisent sur la notion de patrie ${ }^{5}$. La relation pédagogique mise en œuvre s'appuie sur le statut des prêtres au sein des communautés locales. Ils constituent des autorités religieuses de proximité, et sont les principaux détenteurs locaux de la parole publique, à la fois par la messe et par la pratique de l'enseignement scolaire, qui, fréquente dans les paroisses rurales dès la fin du XVIII ${ }^{\mathrm{e}}$ siècle, leur est systématiquement imposée depuis l'époque napoléonienne. Dans un contexte de très forte pratique catholique - l'anticléricalisme est limité à quelques milieux très circonscrits, inspirés par le matérialisme philosophique 6 -, la relation d'autorité a déterminé un processus de politisation descendante, qui s'est ancré dans les espaces et les symboles de la religion. La prédication en a été la principale forme, pratiquée à la fois dans le cadre habituel de la messe et sous la forme de missions itinérantes. L'impulsion que la hiérarchie ecclésiastique méridionale avait donnée à cette pratique au XVIII ${ }^{\mathrm{e}}$ siècle a été réinvestie pour les besoins de la révolution : en 1799, l'historien napolitain Pietro Colletta relève la multiplication des prises de parole politiques des prêtres et des moines, qui justifient la révolution par l'Évangile et multiplient les prophéties politiques en faveur de la liberté

\footnotetext{
${ }^{3}$ Maurizio Isabella, « Religion, Revolution and Popular Mobilization », dans Joanna Innes, Mark Philp (dir.), Re-imagining Democracy in the Mediterranean 1780-1860, Oxford, Oxford University Press, 2018, p. 231-251.

${ }^{4}$ Sur cette notion, voir parmi une riche littérature de sciences sociales Claire Judde de la Rivière, Julien Weisbein (dir.), «Dire et faire le commun. Les formes de la politisation ordinaire du Moyen Âge à nos jours », Politix, n¹19, 2017/3, p. 7-30.

${ }^{5}$ Arianna Arisi Rota, Monica Ferrari, Matteo Morandi (dir.), Patrioti si diventa. Luoghi e linguaggi di pedagogia patriottica nell'Italia unita, Milan, FrancoAngeli, 2009.

6 Gabriele De Rosa, "La vita religiosa nel Mezzogiorno durante la dominazione francese ", dans Bernard Plongeron (dir.), Pratiques religieuses, mentalités et spiritualités dans l'Europe révolutionnaire (1770-1820), Turnhout, Brepols, 1988, p. 104-111.
} 
et de la république7. L'utilisation du lexique religieux, légitimé à la fois par le port de la croix et des vêtements sacerdotaux, constitue donc une garantie donnée à la révolution, jugée capable de susciter le soutien du peuple. Ces pratiques, bien qu'individuelles et isolées, ont retenu l'intérêt des chefs révolutionnaires, qui ont pris en considération leur capacité performative. Parmi d'autres initiatives, la femme de lettres Eleonora Fonseca Pimentel envisage ainsi, dès 1799, des «missions civiles » modelées à l'exemple des missions catholiques, parce qu'elles seraient capables de donner une image rassurante et légitime de l'insurrection ${ }^{8}$.

Le recours à la parole et à l'action des prêtres permet donc de diffuser la révolution auprès du peuple, en l'encadrant par des langages qu'il connaît et qu'il approuve. La révolution de 1820-1821 voit le phénomène s'accentuer ${ }^{9}$, dans des proportions suffisamment notables pour être relevées par des observateurs étrangers. C'est le cas du savant catalan Carles Gimbernat, qui en impute le succès très rapide à « quelques curés, qui ont prêché auprès [du peuple] l'insurrection comme une obligation religieuse ». Il rappelle comment deux d'entre eux, Luigi Minichini et Vincenzo Mirabella, se sont employés à lever des hommes armés pour soutenir la révolution, en entourant la prédication d'un important répertoire symbolique destiné à en exprimer la sacralité. La bénédiction des banderoles révolutionnaires, la plantation de grandes croix d'argent prises des autels en sont les manifestations principales ${ }^{10}$. Ainsi orientée, la parole sacerdotale affirme la sacralité de la révolution, en la plaçant sous l'autorité de la religion. Les messes dominicales, les fêtes religieuses sont l'occasion de mettre en évidence ce lien, en associant des prêches politisés, des symboles religieux et des symboles politiques. C'est par la messe que des prêtres de la province d'Avellino, affiliés à la Charbonnerie, ont prédit l'adoption prochaine de la constitution de Cadix par la monarchie napolitaine dans les premiers mois de 1820, et que d'autres ont expliqué au peuple l'intérêt de ce texte peu après sa promulgation ${ }^{11}$. La même année, le prêtre Nicola Martino, dans le village calabrais de Mormanno, associe le drapeau carbonaro au crucifix et à l'image de la Vierge, pour affirmer, le jour de l'Assomption, que la révolution est

7 Pietro Colletta, Storia del reame di Napoli dal 1734 al 1825, Capolago, Tipografia Svizzera, 1834, p. 225.

${ }^{8}$ L'idée est notamment développée dans le Monitore Napoletano du 5 février 1799. Voir Enrico Francia, «Predicare la rivoluzione. L'oratoria politico-religiosa del Risorgimento », dans Mario Isnenghi (dir.), Pensare la nazione. Silvio Lanaro e l'Italia contemporanea, Rome, Donzelli, 2012, p. 17-27.

9 Pierre-Marie Delpu, «Patriotisme libéral et nation catholique : les prêtres libéraux dans la révolution napolitaine de 1820-1821 », Studi Storici, n58, 2017/3, p. 545-571.

${ }^{10}$ Carles Gimbernat, supplément à la Miscelanea de comercio, artes y literatura, $\mathrm{n}^{\circ} 158,5$ août 1820 , p. 5.

${ }^{11}$ Biagio Gamboa, Storia della rivoluzione di Napoli entrante il Luglio 1820, Naples, Trani, 1820, passim. La province du Principat Ultérieur, autour d'Avellino, a vu s'effectuer les premiers développements de la révolution à partir du $1^{\mathrm{er}}$ juillet 1820. 
placée sous la protection de la figure mariale ${ }^{12}$. La pédagogie assumée par les prêtres libéraux consiste donc à expliciter et à vulgariser les idées fortes de la révolution, tout en les diffusant par répétition à l'office dominical.

La symbolique mise en œuvre révèle des usages sécularisés de la religion, qui transfèrent le lexique et les références culturelles catholiques à l'espace profane de la politique. La place importante des prêtres dans la Charbonnerie napolitaine, depuis le début des années 1810, explique leur forte mobilisation séditieuse et l'exploitation de codes religieux et mystiques, déjà pratiquée par la société secrète ${ }^{13}$. Les prédications prônant l'opposition au roi ont été courantes, mais elles ont relevé d'efforts individuels et spontanés et n'ont pas reçu le soutien de l'institution ecclésiale. L'appui donné aux insurrections, qui s'est maintenu jusqu'à l'unification de l'Italie en 1860, a principalement relevé du bas-clergé, qu'il s'agisse des curés de village ou de quelques ordres monastiques comme les franciscains ou les capucins ${ }^{14}$; à l'inverse, la hiérarchie ecclésiastique a condamné, de façon quasi-unanime, l'implication des prêtres du côté des révolutionnaires. C'est le cas de l'archevêque de Catanzaro Michele Basilio Clary, qui dénonce en 1822 le «libéralisme chrétien» dont une fraction significative des ecclésiastiques méridionaux se seraient faits les porteurs au moment de l'insurrection de 1820-182115. Dès lors, la prédication politique relève d'une initiative autonome et individuelle, liée à la fois à la fonction d'enseignement et au statut d'autorités locales. Sans se limiter au seul espace de l'église, elle s'ancre dans des lieux a priori étrangers à la prédication religieuse, ceux pratiqués par la sociabilité des libéraux. Au printemps et à l'été 1820, Luigi Minichini, curé de Nola en périphérie proche de Naples, prononce des prêches réguliers dans le café Montano, tenu par un vétéran d'Empire et qui est alors l'un des principaux repaires des opposants au roi ${ }^{16}$.Traditionnellement dénoncés dans les discours sacerdotaux comme des lieux de perdition, les cafés sont ici utilisés comme des lieux moteurs de la politisation par leur capacité à rassembler les hommes de la bourgeoisie et des classes moyennes, cible habituelle du courant libéral dans l'Italie du $\mathrm{XIX}^{\mathrm{e}}$ siècle. Dans une société marquée par un fort analphabétisme populaire, les prêtres ont ainsi constitué des passeurs privilégiés des idées révolutionnaires, qui appuient leur rôle de politisation sur les sociabilités ordinaires. Alors que les trois révolutions ont vu

\footnotetext{
${ }^{12}$ Archivio di Stato di Napoli (par la suite ASN), Archivio privato Borbone, 726, 15. La dévotion mariale connaît un usage militant soutenu dans le Mezzogiorno, depuis l'époque moderne, dans le sillage de la Contre-Réforme.

13 P.-M. Delpu, « Patriotisme libéral et nation catholique... », op. cit.

14 Pour les franciscains, voir Alfonso Conte, " "Con il drappo tricolore cinto intorno al saio" : i francescani napoletani nel processo di unificazione », Meridiana. Storia e scienze sociali, 78, 2013, p. 119-133.

${ }_{15}$ Michele Basilio Clary, Il liberalismo cristiano. Omelie sacro-politiche recitate nella sua cattedrale nell'avvento dell'anno 1821, Messine, Pappalardo, 1822.

16 Luca Di Mauro, Le secret et Polichinelle: cultures et pratiques de la clandestinité politique à Naples au début du XIXe siècle, 1799-1821, thèse de doctorat, Université Paris 1, 2015, p. 473. Sur le rôle politique des cafés, voir Mario Isnenghi, L'Italia in piazza. I luoghi della vita pubblica dal 1848 ai giorni nostri, Bologne, il Mulino, 2004, p. 36-37.
} 
se construire un début d'espace public reposant sur la place de l'écrit, à travers l'implication soutenue des libraires et des éditeurs, la prédication politique a été un moyen de les inscrire dans une culture dominante orale et dialectophone. En 1821, Minichini théorise sous le nom d'illustrazione ce processus d'investissement de l'espace public et rappelle que l'initiative en incombe aux clercs, chargés de transmettre et d'interpréter la révolution ${ }^{17}$.

\section{DES INTERPRETES DE LA REVOLUTION}

La prédication politique relève donc d'un usage sécularisé de références religieuses destinées à enseigner et à légitimer la révolution. Dans une société marquée par un très fort taux d'analphabétisme populaire, le recours à l'oralité s'est imposé comme une nécessité pour la conversion politique des masses à la révolution. S'il est difficile de connaître le contenu précis des prêches, notamment parce qu'ils sont très rares à avoir été publiés, à la différence d'autres États italiens de la même époque ${ }^{18}$, on sait par les sources policières et judiciaires que beaucoup d'entre eux ont été l'occasion de lire et d'expliquer des textes politiques écrits auxquels une grande partie des sujets du royaume n'avaient pas accès. À l'été 1848, un prêtre de la baie de Naples, Pietro Palomba, profite de la messe pour donner des lectures publiques de feuilles volantes et de tracts politiques, au nom desquels il exhorte les sujets du village à se mobiliser contre le roi ${ }^{19}$. Les textes, les notions placées au centre de la relation pédagogique relèvent donc de l'initiative des seuls prêtres, sans qu'il y ait d'impulsion réelle pour uniformiser leurs discours. Le royaume a connu, au cours des trois révolutions, des catéchismes patriotiques destinés à être lus par les clercs dans leurs fonctions d'enseignement et de prédication, mais leur contenu a davantage été indicatif qu'il n'a été prescriptif. Ces textes, qui construisent une relation pédagogique indirecte puisqu'ils sont destinés à être lus au peuple par des acteurs instruits, se sont développés dès 1799, alors que la circulation des catéchismes révolutionnaires français a rencontré une pratique locale, celle du catéchisme d'alphabétisation promu par l'évêque napolitain Alphonse de Liguori au XVIII ${ }^{e}$ siècle $^{20}$. En 1799, celui publié par Stefano Pistoia se destine explicitement « aux précepteurs des écoles et spécialement aux curés des campagnes ${ }^{21}$. À partir de 1820, l'intérêt porté aux catéchismes s'est confirmé, alors que la constitution

\footnotetext{
17 Luigi Minichini, Luglio 1820. Cronaca di una rivoluzione, publ. Mario Themelly, Rome, Bulzoni, 1979.

18 Par exemple dans le grand-duché de Toscane et dans l'État pontifical, où les publications de prêches ont été nombreuses en 1848 (E. Francia, "Predicare la rivoluzione... », op. cit.).

${ }^{19}$ ASN, Alta Polizia, 41, 264.

${ }^{20}$ Luciano Guerci, Istruire nelle verità repubblicane. La letteratura politica per il popolo nell'Italia in rivoluzione (1796-1799), Bologne, il Mulino, 1999.

${ }^{21}$ Stefano Pistoia, Catechismo nazionale pel popolo per uso de'parocchi, Naples, s.d., 1799.
} 
de Cadix de 1812, adoptée par le régime révolutionnaire napolitain entre juillet 1820 et mars 1821, avait établi la nécessité de ce type d'écrits. À l'exemple des catéchismes espagnols, qui ont circulé dans le royaume, les propositions se sont multipliées pour établir un texte capable de faire l'objet d'un usage uniforme à l'échelle nationale ; d'autres y ont vu un moyen privilégié d'encourager la vertu publique ${ }^{22}$. Ces textes ont donc été utilisés pour leur valeur d'édification morale et pour leur capacité à produire une éthique propre à la révolution. Ils constituent un emprunt au discours religieux pour les besoins de la politisation des masses. Leur usage pose cependant la question de la matérialité du processus de transmission.

En 1820 et en 1848, les feuilles volantes sont nombreuses à mettre en scène les modalités de la parole politique des prêtres. Elles en font un modèle de communication politique destiné à être investi par les propagandistes de la révolution. Les publications de dialogues fictifs se multiplient, à l'image des chiacchieriate (conversations) et des parlate (textes parlés) de 1820, qui se présentent sous la forme de catéchismes simplifiés faisant alterner des questions et des réponses entre des personnages stéréotypés. S'il s'agit au départ d'allégories, qui mettent en scène de façon symbolique des prêches révolutionnaires à valeur pédagogique, ils intègrent de façon explicite, en 1848, des figures de bons et de mauvais prêtres ${ }^{23}$. À la différence des catéchismes politiques, ces dialogues révèlent l'usage prépondérant des dialectes dans les stratégies de communication utilisées par les prêtres. Les seules chiacchieriate dont on dispose sont en napolitain, mais d'autres initiatives montrent que des dialectes régionaux ont également fait l'objet d'usages politiques, à l'image du calabrais, langue dans laquelle le chanoine de Cosenza Nicola Lepiane avait traduit le catéchisme des carbonari en $1813^{24}$. Ces usages des dialectes ne sont pas nouveaux : ils sont un vecteur de la communication courante, déjà envisagés en 1799 comme un moyen de transmettre les idées révolutionnaires auprès du peuple ${ }^{25}$. Ils font l'objet d'une utilisation continue tout au long du premier XIX $\mathrm{X}^{\mathrm{e}}$ siècle, et relèvent d'une stratégie de politisation autonome. Celle-ci fait intervenir des formes d'oralité multiple: c'est le cas d'Antonio Turano, dans le village calabrais de San Lucido, qui prêche en mars 1848 contre le roi-tyran Ferdinand II, qu'il accuse d'avoir usurpé la propriété privée des sujets du royaume. Armé d'un pistolet et d'un poignard, il engage alors une procession chantée, qui investit l'espace du village au départ de l'église au son d'une « guitare française » en répétant cette strophe :

C'est la fin des Puissances!

22 Par exemple "Catechismo costituzionale pel uso del Regno Unito delle Sicilie», Minerva Napoletana, I, 1, 1820, p. 23-31.

${ }^{23}$ Par exemple Biblioteca di Storia Moderna e Contemporanea, Rome (BSMC), Fo. Ris. III.A.17, 116, 116, Dialogo di un parroco ed un suo filiano sulla Costituzione data da S.M. nel 29 gennaio 1848, Naples, s.n., 26 février 1848, et BSMC, Bandi, B2, 343, B2, 343, Parole di un sacerdote a'suoi fratelli ecclesiastici napoletani, Naples, Nobile, 5 mars 1848. Pour les chiacchieriate de 1820-1821, voir ASN, Archivio privato Borbone, 2035.

${ }^{24}$ Davide Andreotti, Storia dei Cosentini, Cosenza, Migliaccio, 1864, t. III, p. 204.

25 Domenico Scafoglio, Lazzari e giacobini. Cultura popolare e rivoluzione a Napoli nel 1799, Naples, L'Ancora, 1999. 
C'est la fin des Excellences !

Un seul instant triomphera,

La liberté qui toujours vivra !26

Plusieurs témoignages, recueillis lors du procès politique intenté à Turano au lendemain de la révolution, signalent le succès momentané de la chanson, y compris dans les villages voisins, au point qu'elle serait devenue un slogan des révolutionnaires locaux. L'usage de la chanson rejoint une pratique ancrée dans la culture populaire italienne du XIXe siècle, qui en fait un outil courant de mobilisation des masses, très pratiqué en $1848^{28}$. La pratique du prêche chanté, qui emprunte au répertoire des cantiques, est donc détournée et laïcisée pour les besoins de la révolution.

La performativité de la parole sacerdotale s'explique donc à la fois par l'utilisation des formes ordinaires de l'oralité et par leur encadrement rituel. Elles sont un moyen privilégié d'intégrer les masses, parce qu'elles font appel à des émotions collectives à valeur mobilisatrice. En 1848, l'évocation de la sacralité de la révolution, l'image du «pape national et libéral» Pie IX, qui a soutenu les révolutions italiennes de 1848 à leurs débuts, sont des arguments régulièrement invoqués, à l'appui de rites et d'images empruntés à la religion. Dans les premiers mois de la révolution, l'archiprêtre Antonio Greco prononce des éloges publics du pape et fait circuler rituellement son buste dans les rues de Catanzaro ${ }^{29}$. Au même moment, dans le village calabrais de Cerisano, le prêtre Luigi Greco en appelle à la ferveur religieuse de ses ouailles pour les inciter à soutenir la révolution :

Vous, habitants de Cerisano, soyez citoyens, libres, Chrétiens! Quoi d'autre nous commande notre religion, quoi d'autre que l'amour et la liberté. [...] Prions pour nos frères d'Italie, qu'ils brisent leurs chaînes! Par Dieu, mes frères, la main sur l'autel, jurons de nous défendre jusqu'à notre dernière goutte de sang! Mais avant toute chose jurons d'être Italiens, d'être fidèles au Roi et à la Constitution ${ }^{30}$.

L'appel à la mobilisation armée et au sacrifice constitue l'élément principal de cette rhétorique, qui donne à voir des différences de degré, voire de nature, entre des prises de positions plus modérées et d'autres plus radicales. Cette diversité se retrouve dansles évocations des figures de martyrs populaires, sacrifiées par la monarchie pour la liberté du royaume, dont le souvenir est régulièrement rappelé au sujet du royaume lors des prêches patriotiques. Celui-ci est doté d'une charge émotive forte: les martyrs sont destinés à être admirés et célébrés sur le modèle des martyrs du christianisme. Mais par rapport à ce modèle religieux, les références politiques au sacrifice ont la particularité

\footnotetext{
${ }^{26}$ Archivio di Stato di Cosenza (ASC), Processi politici, 41, 250.

${ }^{28}$ Antonio Buttiglione, La Rivoluzione in "periferia". Movimenti popolari e borghesia rossa nelle Due Sicilie (1830-1848), thèse de doctorat, Università degli Studi della Tuscia, 2018, p. 95. Sur l'usage de la chanson politique dans l'Italie de 1848, voir Michele Toss, «I luoghi e gli usi della canzone sociale in Italia (1848-1870) », dans Antonio Carlini (dir.), Accademie e società filarmoniche in Italia. Studi e ricerche, Trente, Società filarmonica del Trento, 2012, p. 159-219.

${ }^{29}$ ASN, Archivio privato Borbone, 1044, 38.

${ }^{30}$ Prêche retranscrit dans Il Calabrese rigenerato, VI, 5, 15 mars 1848, p. 2.
} 
d'appeler à la vengeance, élément fortement mobilisateur qui prend notamment la forme d'appels à la croisade, désormais dirigée contre des adversaires chrétiens, qu'il s'agisse des tyrans ou de l'armée autrichienne qui a réprimé les premiers élans révolutionnaires dans d'autres États italiens. C'est donc une valeur empruntée à la religion, la fraternité italienne, qui est mobilisée pour appeler aux armes. En mars 1848, le frère dominicain Raffaele Orioli appelle les habitants de Cosenza à "gagner la couronne du martyre » et annonce la venue du «temps de la dernière croisade »31. Il recourt ainsi à un modèle historique, celui de l'appel pontifical à la croisade, dans un contexte où une grande partie du clergé est identifié à la figure du pape Pie IX. Les prêches politiques contribuent donc à poser les bases d'une éthique révolutionnaire modelée à l'exemple de la religion et capable de produire l'identification massive des sujets du royaume. Ils font l'objet d'usages multiples, de plus en plus radicaux, et d'initiatives visant à en améliorer l'efficacité.

\section{PERFECTIONNEMENT ET RADICALISATION DE LA PAROLE SACERDOTALE}

Envisagée initialement comme une voie possible de la politisation des masses, la parole sacerdotale est progressivement devenue une pratique formalisée, capable de constituer un outil de pédagogie patriotique efficace. Si en 1799 et en 1820, les prêches révolutionnaires ont relevé d'initiatives individuelles et spontanées, la rhétorique sacerdotale s'est en partie perfectionnée dans les années 1830 et 1840, dans le cadre des écoles de rhétorique napolitaines. Alors que les acteurs de l'opposition au roi envisagent de plus en plus systématiquement les moyens d'intégrer le peuple à la révolution, le prêtre calabrais Paolo Pellicano, ordonné à Reggio en 1836, se rend à Naples pour suivre les cours d'éloquence donnés par le grammairien napolitain Basilio Puoti, tenant du " purisme » littéraire et référence commune d'une grande partie de la génération des révolutionnaires méridionaux de 1848. De retour à Reggio en 1838, Pellicano met en pratique les formes d'éloquence étudiées à Naples, les enseigne à son tour au séminaire provincial et leur consacre plusieurs articles dans la revue libérale locale, la Fata Morgana, dont il est l'un des principaux rédacteurs. Lui-même formé par le prêtre républicain Giuseppe Battaglia dans les années 1810 et conscient du rôle déterminant joué par le bas-clergé local dans la diffusion de la révolution, il développe l'intérêt de l'éloquence sacerdotale pour la diffusion des idées libérales et incite les prêtres du diocèse à s'y former ${ }^{32}$. L'initiative de Pellicano relève d'un double transfert, qui exporte

\footnotetext{
${ }^{31}$ Raffaele Orioli, « Proclama », Il Calabrese rigenerato, VI, 1, 15 février 1848, p. 4. Sur le martyre politique, voir Pierre-Marie Delpu, «Une religion politique. Les usages des martyrs révolutionnaires dans le royaume des Deux-Siciles (années 1820-années 1850) », Revue d'histoire moderne \& contemporaine, ${ }^{\circ} 64,2017 / 1, \mathrm{p} .7-31$.

32 Paolo Pellicano, «I cappuccini di Reggio », La Fata Morgana, I, 20, 1 er février 1839, p. 1 ; Id., « Annunzio », Ibid., I, 21, 15 février 1839, p. 1.
} 
en province des techniques rhétoriques acquises à Naples et qui transfère dans l'ordre politique des compétences littéraires. Mais elle demeure isolée: il est difficile d'en mesurer l'impact réel sur les prêtres du diocèse, et dans les autres provinces, la parole politique des prêtres continue de suivre les schémas traditionnels.

Si les formes rhétoriques utilisées sont évolutives, la parole sacerdotale recouvre des attitudes politiques de plus en plus diverses. Elle a d'abord servi à légitimer la révolution, à lui donner un crédit moral et rassurant, à justifier la constitution à donner au royaume en en rappelant la sacralité. Elle exprime donc initialement des positions politiques modérées, et ne connaît que ponctuellement des usages plus radicaux, tels ceux du prêtre calabrais Nicola Martino qui, en 1820, se posait en expiateur et en rédempteur des provinces calabraises contre la monarchie des Bourbons qu'il jugeait tyrannique ${ }^{33}$. Mais alors que se recompose le paysage politique méridional dans les années 1830, le succès du courant démocrate alors émergent, en particulier dans les périphéries du royaume, explique la radicalisation de plus en plus fréquente des prêches patriotiques. D'abord circonscrite à des sociétés secrètes et à des associations chrétiennes, dont la Società Evangelica, fondée en 1841, est la plus significative, elle s'est par la suite généralisée lorsqu'est intervenu le tournant conservateur du régime révolutionnaire à partir d'avril 1848. Les massacres perpétrés par l'armée bourbonienne sur des civils le 15 mai 1848 le confirment, et expliquent des appels de plus en plus fréquents à la vengeance et au sacrifice personnel. Dans la ville calabraise de Castrovillari, lorsque le prêtre Michele Bellizzi célèbre une messe en l'honneur des martyrs du 15 mai, il appelle les habitants de la paroisse à s'armer pour tuer le roi et à utiliser son sang comme engrais pour faire pousser des arbres de la liberté ${ }^{34}$. Début juin, deux prêtres de Cosenza baptisent un bélier et une vache des prénoms du roi et de la reine, les abattent et les font rôtir pour les servir à la table d'un banquet protestataire ${ }^{35}$. Ces actes sacrilèges, qui accompagnent des discours vengeurs, sont révélateurs de l'évolution tyrannicide et expiatrice des discours sacerdotaux. Ils relèvent moins d'une idéologie républicaine assumée que d'une opposition spontanée aux dérives jugées tyranniques de la monarchie bourbonienne. Le martyre et la croisade continuent d'être les principales références invoquées, mais elles connaissent une inflexion significative qui les associe à l'horizon de la violence politique, alors qu'elles sont également utilisées par des partisans laïques de la révolution. Elle se retrouve dans les gestes et les objets associés au prêche, de plus en plus souvent prononcé par des prêtres en armes qui appellent leurs ouailles à s'engager à leurs côtés. Dès lors, l'appel à s'armer pour partir en croisade devient un appel à la résistance armée contre la monarchie. En Calabre encore, les prêtres radicaux sont nombreux à prêcher le départ pour les camps de révoltés, structures politiques improvisées dans lesquelles des révolutionnaires locaux organisent l'opposition au roi en regroupant ses acteurs à grande échelle, pour protéger les populations locales des possibles menées de l'armée bourbonienne dans la province.

\footnotetext{
33 ASN, Archivio privato Borbone, 726, 14.

${ }^{34}$ ASN, Archivio privato Borbone, 1044, 35.

35 ASN, Alta Polizia, 640, 2563.
} 
Les prêtres ont ainsi contribué à construire les radicalités révolutionnaires, exploitant à la fois des motivations socio-économiques et le mécontentement de plus en plus fréquent des sujets contre la monarchie. Le départ vers les camps de révoltés est souvent présenté comme une impulsion collective, qu'ils ont cherché à orienter par le prêche et qu'ils ont accompagné d'actes symboliques. Les prédications régicides ont donné lieu à des destructions ritualisées de statues du couple royal, sous la forme d'exécutions symboliques, à la suite de jugements simulés. Dans le village calabrais de Saracena, le prêtre Antonino Prioli, qui a déjà participé à la révolution de 1820-1821 en tant que carbonaro, lit une sentence d'exécution aux deux statues de grès du roi et de la reine, qu'il a exposées sur la place du village, avant de les fusiller devant la foule des habitants de la commune ${ }^{36}$. Ces pratiques, qui empruntent aux charivaris sur lesquels s'est couramment appuyée la justice populaire dans la première moitié du XIX ${ }^{\mathrm{e}}$ siècle, sont pourtant envisagées par les autorités comme de simples formes de vandalisme, sans saisir la réalité de leur signification politique. La prise en charge de la justice populaire par les prêtres eux-mêmes permet cependant d'encourager la révolte locale contre la monarchie. Dans d'autres cas, le prêche se limite à appeler les sujets du roi à détruire eux-mêmes les statues : plusieurs villages de la province de Reggio en donnent des exemples, dans le cadre des résistances de l'été $1848^{37}$. Le sacrilège, la vengeance sont révélateurs des stratégies de mobilisation utilisées par les prêtres du royaume : ils montrent à la fois la destruction et le transfert de l'autorité centrale vers les communautés locales, et contribuent à l'ampleur prise par les résistances populaires.

Le développement d'une parole politique capable de mobiliser des émotions collectives et fortement encadrée par des gestes et des objets à valeur politique pose la question de la performativité des prêches révolutionnaires. En 1820, ils ont donné lieu à des impasses : les feuilles volantes en circulation dans la capitale du royaume montrent que les prédications n'ont pas toujours été comprises. Alors qu'il s'agissait d'expliquer au peuple des notions politiques méconnues, elles révèlent la difficulté pour une partie des habitants du royaume à comprendre des mots qui n'ont pas leur équivalent en dialecte :

- S. [Sebeto] Alors, dis-moi, ils disent bien Viva u Re, Viva a Costetuzione !

- CdN [Corpo di Napoli] Mais alors, c'est vrai ! Il me semble bien qu'ils disent viva a custipazione, viva a conclusione 38 .

- G. [Giagante] Que veux-tu dire ? Constitution?

36 ASN, Archivio privato Borbone, 1044, 38. Sur l'iconoclasme comme pratique de contestation politique, voir Arianna Arisi Rota, " "Così brutale insulto". Gesti iconoclasti nella peninsola italiana tra 1848 e seconda Restaurazione », Memoria e Ricerca, 2018/1, p. 61-76.

37 Par exemple à Fiumara et à San Luca : voir Archivio di Stato di Reggio Calabria (ASRC), Atti di Polizia, I, 1, 81 et 252.

${ }^{38}$ ASN, Archivio privato Borbone, 2035, 1. Le Sebeto (nom du fleuve qui irriguait Naples dans l'Antiquité) et le Corpo di Napoli désignent les deux interlocuteurs les plus fréquents de la chiacchieriata ; le premier est docte, le second est ignorant. 
- CdN. Costicazione, costsuzione, voilà39.

Ces incompréhensions se retrouvent en 1848. Dans la province de Reggio, certains prêches ont suscité des moqueries de la part des populations locales : le chroniqueur Antonio Bonafede rappelle que le mot d'ordre Viva Pio IX, viva l'Italia, viva la Costituzione a parfois été déformé en viva Pinomo, viva la Calia, viva la Costituzione 40 . Plus que l'invocation des mots d'ordre et des notions politiques, c'est la référence aux exactions de la monarchie et à la situation économique des habitants du royaume qui a été la plus mobilisatrice : elle explique des initiatives, apparues à l'été 1848, consistant à inciter des sujets à partir en croisade ou dans les camps de révoltés contre la promesse d'une somme d'argent. Dans des provinces marquées par une pauvreté structurelle, renforcée par les conséquences de la crise économique européenne de 1847, l'argument s'est révélé fortement mobilisateur. Dans le village de Galatro, près de Reggio Calabria, le prêtre Giuseppe Galloro dit pendant la messe avoir volé une valise d'argent appartenant au chef des armées bourboniennes en Calabre, et promet de l'utiliser pour distribuer trois carlins par jour à ceux qui le suivront dans les camps de révoltés ${ }^{41}$. Les attestations de cette pratique sont nombreuses, et sont apparues efficaces: la participation à la résistance armée de journaliers agricoles calabrais ou salernitains, de petits artisans des quartiers modestes de Naples a été réelle, même si elle demeure difficile à quantifier. Mais la nécessité de subventionner la résistance est révélatrice des limites de la prédication politique, qui a peiné à convaincre massivement les classes populaires.

Parce qu'elle s'appuie sur une relation pédagogique empruntée aux pratiques sociales ordinaires, la parole sacerdotale s'est donc imposée, au cours du premier XIX ${ }^{\mathrm{e}}$ siècle napolitain, comme l'un des outils les plus puissants d'acculturation politique à destination du peuple. L'utilisation d'images, de symboles, de notions empruntées au répertoire religieux ont facilité l'identification des populations méridionales à la cause révolutionnaire et ont permis leur entrée massive en politique, dont l'ampleur des résistances armées de l'été 1848 est révélatrice. Mais les références religieuses servent surtout à identifier les combats politiques aux valeurs chrétiennes et à montrer la sacralité de la révolution: parce qu'elles sont l'objet d'emplois sécularisés, elles constituent des « religions politiques » à valeur légitimatrice, qui contribuent à poser les

39 ASN, Archivio privato Borbone, 2035, 8. Le Géant (Giagante) est une variante du Sebeto. Les deux termes en dialecte utilisés sont de simples déformations lexicales, qui ne désignent rien de précis en napolitain.

${ }^{40}$ Antonio Bonafede, Sugli avvenimenti de'Fratelli Bandiera e di Michele Bello in Calabria, Naples, s.n., 1848, p. 67. Le terme Pinomo est une simple déformation phonologique de l'italien Pio Nono (Pie IX). Le terme calia désigne une futilité ou une personne ennuyeuse.

${ }^{41}$ ASRC, Atti di Polizia, I, 1, 175. 
bases de ce que sera le répertoire intégrateur du Risorgimento ${ }^{42}$. Les religions politiques ne sont donc pas une exception propre à l'Italie méridionale : elles s'inscrivent dans une évolution plus large propre aux sociétés de l'Europe méditerranéenne, qui permet leur entrée dans les logiques de la politique dite «moderne » au cours de la période 1800$1850^{43}$. Ce sont plutôt l'ampleur du phénomène et le sens des références utilisées qui constituent une singularité du Mezzogiorno, même s'ils sont parfois mobilisés par des acteurs plus divers. En 1820, ce sont ainsi des artisans de la ville de Naples qui deviennent les protagonistes de certaines chiacchieriate en lieu et place des prêtres ${ }^{44}$. Loin de se limiter aux seuls partisans de la révolution, ce répertoire fait l'objet d'usages politiques plus diffus, et relève plutôt d'un ressort ordinaire de la mobilisation des masses que d'une particularité liée à l'idéologie de la révolution. L'évolution du royaume après son intégration à l'Italie unie l'illustre : lorsque se déploie un vaste mouvement de brigandage en réaction au nouvel État, ce sont encore les prêtres qui jouent un rôle déterminant dans l'affirmation des attitudes politiques, entre soutien au brigandage et perpétuation de la tradition libérale méridionale ${ }^{45}$.

42 Dans un livre devenu classique, Alberto Mario Banti rappelle que la sainteté, empruntée au répertoire religieux, constitue l'une des trois valeurs fondatrices du Risorgimento. Voir La nazione del Risorgimento. Parentela, santità ed onore alle origini dell'Italia unita, Turin, Einaudi, 2000.

43 Sur cette évolution, voir Jean-Philippe Luis, "Considérations pour une histoire de la naissance de la politique moderne en Espagne », Mélanges de la Casa de Velázquez, n³4, 2005/2, p. 15-26. La notion, bien étudiée pour le cas espagnol, l'est encore peu pour les États de la péninsule italienne.

${ }^{44}$ C'est le cas du cordonnier Masto Nnicola et du barbier Cuosemo, mis en scène dans une série de chiacchieriate de Paolo Giaramicca (ASN, Archivio privato Borbone, 2035). Les mêmes personnages sont réutilisés dans la propagande révolutionnaire de 1848.

45 Par exemple et pour la Basilicate du début des années 1860, voir Pierre-Yves Manchon, Guerre civile et formation de l'État dans le Midi d'Italie des lendemains de l'Unité (1860-1865). Histoire et usages du « Grand Brigandage » en Basilicate, thèse de doctorat, Université Paris 1, 2013, chapitre 2. 